\title{
Vaccination Recommendations in Patients with Autoimmune inflammatory Rheumatic Diseases
}

\author{
Y.A.Abd El-Hamid, M.E.Yousef and S.M.Rashad \\ Rheumatology, Rehabilitation and physical medicine, Dept., Faculty of Medicine, Benha Univ., Benha, Egypt \\ E-Mail:samar@gmail.com
}

\begin{abstract}
The quantity of people with immune system fiery rheumatic sicknesses (AIIRDs) treated with immunosuppressive medications is expanding consistently. The assortment of immunosuppressive medications and, specifically, natural treatments is likewise rising. The immunosuppressants, just as the AIIRD itself, increment the danger of disease in this populace. In this way, forestalling diseases by methods for inoculation is of most extreme significance. New Swiss immunization suggestions for AIIRD patients were started by the Swiss Federal Office of Public Health and arranged by a working gathering of the Federal Commission for Vaccination Issues just as by meeting of global specialists.; A writing search was acted in electronic data sets (Cochrane, Medline, PubMed, Embase). Furthermore, unpublished writing was recognized through a focused on site search of significant associations and global meetings managing inoculation, irresistible illnesses and rheumatology.; Although information are scant, the accompanying central matters were recovered from the writing. Inactivated immunizations are protected, however their immunogenicity might be diminished in AIIRD patients, particularly in the event that they are under immunosuppressive treatment. Rituximab and abatacept seem to decrease altogether safe reactions after inoculation. Live immunizations are by and large contraindicated under immunosuppressive treatment attributable to wellbeing concerns. Explicit special cases, just as time stretches for the organization of live immunizations after interference of an immunosuppressive treatment, have been detailed in this article.; More proof with respect to the immunogenicity and wellbeing of inoculations in AIIRD patients under different treatments is required.
\end{abstract}

Keywords: Vaccination, Autoimmune inflammatory rheumatic diseases, Immunosuppressive drugs.

\section{Introduction}

Antibodies are among the best accomplishments of current medication. The best method to forestall irresistible infections in any age bunch is inoculation. In addition, the frequency and weight of antibody preventable sicknesses are expanding. Albeit considered as just youth sicknesses for a long time, right now, antibody preventable contaminations may cause genuine horribleness and mortality in grown-ups and old [1].

Rheumatic issues are among the most predominant ongoing illnesses of the musculoskeletal framework and connective tissue, and they can influence a wide scope old enough gatherings. The predominance of rheumatic infections in everyone goes from $9.8 \%$ to $33.2 \%$, and it has been assessed that $15-45 \%$ of essential consideration doctor counsels are for musculoskeletal issues [2].

Including countless joint inflammation and immune system illnesses, rheumatic problems can influence the bones, joints, and different parts of the musculoskeletal framework, causing horribleness or handicap with resultant medical care use [3].

Rheumatic issues are principally liable for a powerlessness to work and exiting the workforce, a reality which features their gigantic social and monetary effect [4].

Regular rheumatic problems incorporate rheumatoid joint inflammation, fundamental lupus erythematosus, polymyalgia rheumatic, goliath cell arteritis, osteoarthritis, precious stone joint inflammation, paraneoplastic conditions, summed up torment disorder, osteoporosis, seronegative spondyloarthropathy, foundational sclerosis and gout [5].

Patients with rheumatic infections are uniquely shown for immunization. Irresistible infections have expanded the horribleness and mortality among those patients because of related inconveniences, concomintant constant illnesses, immunosuppressive and immunomodulatory drug use, successive hospitalization, and medical procedure. Rheumatic illnesses increment the danger of contamination as well as the danger for a more serious course. Various elements influence the adequacy of vac-cines regulated to patients including the kind of immunization, immunosuppressive immunomodulatory treatment, infection action, age and presence of constant sicknesses [1].

An examination including 46,000 patients with rheumatoid joint inflammation and coordinated with controls revealed that the difficulties of flu were expanded 2.75 folds in patients with rheumatoid joint pain than in those without the condition [6], and this danger has been accounted for to be autonomous of the medication utilized. The flu antibody has been appeared to lessen the seriousness of the infection or assault rate paying little heed to the treatment given [7].

Besides, the actual contamination and the end of immunosuppressive treatment in the time of disease can prompt intensifications of the immune system sickness. The contaminations that are normal and cause difficulties in rheumatic infections are usu-partner antibody preventable sicknesses like flu, intrusive pneumococcal illnesses, lockjaw, diphtheria, herpes zoster [8].

Numerous examinations researched whether immunizations lead to the exacerbation of existing immune system sicknesses have illustrated $[9,10]$. As per Tanriover et al. [8], the inoculation status of a patient with rheumatic infection ought to be looked into following the determination. Any missing immunizations ought to be directed. Serological tests ought to be 
performed to survey the invulnerable status of the patient if there are no inoculation records or if the immunization history is unclear.

The point of the ebb and flow work was to survey and shed a few lights on connection among inoculation and rheumatic infections.

\section{Vaccination in Patients with rheumatic sicknesses}

Inoculation is for the most part viewed as a protected, effective and minimal effort strategy for forestalling certain contaminations. Be that as it may, immunization might be less effectual in (subgroups of) patients with AIIRD, because of their immunosuppressed state [11].

The European League Against Rheumatism (EULAR) assists specialists in arranging inoculations for patients with AIIRD, and toward the finish of 2019 refreshed their proposals [12].

In view of the EULAR rules, the rheumatological group ought to evaluate patients with AIIRD consistently for inoculation status and signs for their update or continuation. A particularly individualized inoculation program ought to be deliberately disclosed to the patient and executed in participation with an essential consideration doctor and a rheumatological group [12].

The best methodology is to manage antibodies during "consistent express", an abatement of AIIRD before arranged immunosuppression (particularly before treatment diminishing B cell checks). For this patient gathering, inoculations ought to be arranged in any event a half year after and a month prior to the following treatment cycle. In situations where this time stretch is unimaginable, vaccination might be considered as a component of the B-cell decrease treatment, considering the likely non-ideal reaction to the antibody. Restricted information about the security and immunogenicity of immunizations during dynamic infection makes a contraindication. It merits stressing that, in serious cases, essential inoculations ought not be deferred [13].

Immunosuppressants used to treat AIIRD incorporate corticosteroids, traditional manufactured illness altering against rheumatic medications, like methotrexate (MTX), sulfasalazine, leflunomide and hydroxychloroquine. Live antibodies are generally contraindicated in seriously immunocompromised patients because of conceivable vaccineinduced infection from viral replication [14].

\section{Discussion}

Patients with AIIRD on Immunosuppressants can securely get inactivated, "executed" antibodies. Information from clinical examinations affirmed that organization of the immunizations against flu, pneumococci, lockjaw pathogen, hepatitis B (HBV), hepatitis A (HAV), and human papillomavirus (HPV) is viable and protected among those patients [12].

Albeit the organization of live, lessened immunizations during immunosuppression ought to be maintained a strategic distance from in patients with AIIRD in light of the fact that live weakened microorganisms can conceivably cause disease, there is a chance of cautious utilization of the measles, mumps, and rubella (MMR), and herpes zoster antibodies. In light of their security information, they can be considered in individuals with AIIRD with a low level of immunosuppression and a high possibility of contracting measles (voyagers) or herpes zoster (hazard gatherings) [15].

In the general evaluation, in a populace of patients with AIRD, antibodies are protected and immunogenic. Methotrexate and glucocorticoids have no plainly unfavorable impact on the immunogenicity of immunizations. Treatment of biologics may speed up the reduction in immune response levels after some time, albeit most of patients during the primary time frame after immunization were suitably gotten [16].

\subsection{Influenza Vaccine}

All immunocompromised AIIRD patients who get the flu antibody interestingly ought to get two immunization dosages, in any event a month separated and afterward one portion yearly on a continuous premise [17]. Flu inoculation ought to happen quite far from a portion of RTX [18].

\subsection{Streptococcus pneumoniae Vaccine (Pneumococcus)}

Pneumococcal inoculation is suggested for all $\geq 65$ year olds. Likewise, immunosuppressed grown-ups ought to get $23 \mathrm{vPPV}$ at age 18 or 5 years after the latest portion, with up to two additional dosages 5 years separated. Recently determined to have a condition requiring immunosuppression and immunization credulous ought to get $13 \mathrm{vPCV}$ followed by $23 \mathrm{vPPV}$ after at any rate two months. The portion of $23 \mathrm{vPPV}$ ought to be rehashed in 5 years and a third portion allowed at 65 years (or 50 years in native individuals) [17].

Preferably, pneumococcal immunization ought to be given before commencement of DMARDs (particularly MTX) [19].

\subsection{Hepatitis A Vaccine (HAV)}

Inoculation ought to be considered on the whole patients as HAV might be serious in the immunocompromised. Two portions of immunization a half year separated are ordinarily suggested: the main portion preceding travel gives satisfactory insurance in sound people.

A subsequent portion helps the resistant reaction to give long haul insusceptibility [17].

\subsection{Hepatitis B Vaccine (HBV)}

Immunization for $\mathrm{HBV}$ is suggested when the patient is at higher danger of serious sickness, for example on immunosuppression, the danger of contracting $\mathrm{HBV}$ is expanded, for example travel to, or home in nations endemic for $\mathrm{HBV}$, there is expanded danger of openness or demonstrated openness to HBV, for example medical care experts, contaminated relative or contacts and in conclusion when defensive HBV antibodies are missing [17]. 


\subsection{Human papilloma infection Vaccine}

There are variable public rules in regards to HPV inoculation [9]. The Australian Technical Advisory Group on Immunization (ATAGI) suggests HPV inoculation for immunocompromised grown-ups paying little heed to age [17].

Youths with AIIRD who have not yet been inoculated might be offered 'get up to speed' as they might be bound to create determined HPV contamination, which may advance to HPV-related illness [20].

Patients with foundational lupus erythematosus (SLE), ought to specifically get immunization against human papillomavirus (HPV) as suggested for everyone, in light of the fact that a large portion of the proof in regards to HPV the study of disease transmission in patients with rheumatic illnesses depends on examinations in ladies with SLE [21].

\subsection{Herpes zoster Vaccine}

All patients matured 50-80 years ought to be considered for $\mathrm{HZ}$ inoculation prior to starting tsDMARDs or bDMARDs. Likewise, those being treated with a TNFi ought to be inoculated after conversation with an expert in regards to the dangers and advantages of immunizing with a live immunization [17].

\subsection{Lockjaw pathogen immunization}

Rituximab regulated 24 weeks before immunization didn't influence reaction to the lockjaw pathogen antibody in patients with RA [22]. A RCT in 54 patients with RA on MTX who began tociluzimab 3 weeks before lockjaw pathogen immunization, and 27 RA MTX illness controls, showed no distinction in immunogenicity of the lockjaw pathogen antibody between gatherings. Notwithstanding, there were just three patients in the tocilizumab + MTX bunch who didn't have a seroprotective immune response level at benchmark. Two out of these three patients arrived at a defensive level 5 weeks after immunization [22].

An observational investigation on immunogenicity of pneumococcal, lockjaw pathogen and H. influenzae type B immunization in 73 patients with SLE detailed a pattern towards a lower reaction in patients on glucocorticoids and azathioprine, which was not indicated for lockjaw pathogen inoculation [11].

\subsection{Bacillus Calmette Guérin Vaccine (BCG)}

The suggestions in regards to the BCG immunization, which is not, at this point suggested in patients with AIIRD, a way to deal with hyposplenic/asplenic patients and to explorers with AIIRD were precluded from the current form. All things being equal, a different thing with respect to the yellow fever immunization in patients making a trip to endemic zones was added [22].

It isn't prescribed in immunocompromised patients because of the conceivable, however generally safe of scattered disease post-immunization [11]. This okay likewise happens following intravesical BCG as therapy of bladder malignancy, which can be related with responsive joint pain [23).

\subsection{Diptheria, lockjaw and pertussis Vaccine}

Grown-ups matured $\geq 50$ years without a promoter portion of diptheria and lockjaw pathogen in the first 10 years ought to get the dTpa (diphtheria-lockjaw pertussis acellular) detailing, which will likewise ensure against pertussis. This contrasts from the DTPa definition, which is utilized in youngsters and contains more modest measures of antigen [17].

\subsection{Measles, mumps and rubella Vaccine (MMR)}

The MMR immunization is a live readiness and not suggested in immunosuppressed patients. Post-openness prophylaxis for non-safe uncovered grown-ups ought to be attempted with typical human immunoglobulin directed inside 6 days of openness [17].

\subsection{Tickborne encephalitis inoculation}

In a past report, in regards to just patients $\geq 60$ years old, it appeared to be that a lower level of those treated with a mix of MTX and against TNF arrived at seroprotective enemy of TBE levels than those treated with MTX or hostile to TNF alone (25\% versus $38 \%-$ 40\%) [11].

\subsection{Salmonella typhi}

Parenteral polysaccharide immunization is prescribed preceding travel to endemic regions. The oral live lessened immunization is contraindicated in the immunocompromised [17].

\subsection{Polio}

One sponsor immunization with inactivated polio antibody (Salk) is exhorted in adulthood, if not previously got. The live lessened (Sabin) immunization is contraindicated in the immunocompromised [17].

\subsection{Meningococcus}

Inoculation with a quadrivalent immunization is suggested for those meeting the sub-Saharan African 'meningitis belt' and is a prerequisite for voyagers to Mecca, Saudi Arabia. The meningococcal quadrivalent form antibody (4vMenCV) is related with preferred immunogenicity over the polysaccharide immunization and is suggested for immunocompromised patients [17].

\subsection{Rabies}

Immunization is suggested following a danger appraisal in regards to the probability of creature openness. Explorers ought to evade contact with canines, felines, monkeys and bats. Any twisted ought to be washed with sudsy water and disinfectant. Post-openness inoculation and utilization of human rabies immunoglobulin ought to be examined with an ID doctor [17].

\subsection{Yellow fever}

No genuine fundamental unfriendly occasions of yellow fever immunization were accounted for in patients with AIIRD. As the immunization has been contraindicated in immunocompromised patients for quite a long time, quantities of studies and included patients are anyway exceptionally low and, as expressed, most patients were revaccinated. Of note, deadly results of individual fever inoculation have been accounted for in immunocompromised patients, incorporating a female 
patient with RA and SLE, who was conceivably treated with glucocorticoids and MTX [25].

\subsection{Cholera}

Organization ought to be considered in the immunocompromised as they have a higher danger of diarrhoeal illness, particularly if intending to visit a region with endemic/plague cholera [17].

\section{Conclusion}

Adverse events of vaccination were generally mild and the rates were comparable to those in healthy persons. Vaccination did not seem to lead to an increase in activity of the underlying AIIRD, but insufficient power of most studies precluded arriving at definite conclusions. The number of studies investigating clinical efficacy of vaccination is still limited.

\section{References}

[1] M.D.Tanrı̈̈ver, S.Akar, N.Türkçapar, Ö.Karadağ, İ.Ertenli, S.Kiraz. "Vaccination recommendations for adult patients with rheumatic diseases. European journal of rheumatology.vol. 3(1),pp. 29-35,2016.

[2] F.Salaffi, M.Di Carlo1, M.Carotti, S.Farah, A.Ciapetti, and M.Gutierrez. "The impact of different rheumatic diseases on healthrelated quality of life: a comparison with a selected sample of healthy individuals using SF-36 questionnaire, EQ-5D and SF-6D utility values." Acta Biomed.vol. 89(4),pp. 541-557,2018.

[3] M.Jokar, and M.Jokar. "Prevalence of Inflammatory Rheumatic Diseases in a Rheumatologic outpatient clinic: analysis of 12626 cases." Rheumatology Research.vol. 3(1),pp. 21-27,2018.

[4] J.C.Branco, A.M.Rodrigues, N.Gouveia. "Prevalence of rheumatic and musculoskeletal diseases and their impact on health-related quality of life, physical function and mental health in Portugal: results from EpiReumaPt- a national health survey.” RMD Open.vol.2(1),pp. e000166,2016.

[5] P.Dhaon, and S.Tripathy. "Rheumatic disease in elderly population, how different from the conventional presentations?" Internet Journal of Rheumatology and Clinical Immunology.vol.3,pp.10,2016.

[6] W.A.Blumentals, A.Arreglado, P.Napalkov, and S.Toovey. "Rheumatoid arthritis and the incidence of influenza and influenza-related complications: a retrospective cohort study." BMC Musculoskelet Disord.vol.13,pp.158,2012.

[7] T.Kobashigawa, A.Nakajima, A.Taniguchi, E.Inoue, E.Tanaka, and S.Momohara. "Vaccination against seasonal influenza is effective in Japanese patients with rheumatoid arthritis enrolled in a large observational cohort." Scand J Rheumatol.vol. 42,pp. 445-450. ,2013.

[8] M. D.Tanrı̈̈ver, S.Akar, N.Türkçapar, Ö.Karadağ, İ.Ertenli, S.Kiraz. "Vaccination recommendations for adult patients with rheumatic diseases European journal of rheumatology.vol. 3(1),pp 29-35,2016.

[9] S.van Assen, O.Elkayam, N.Agmon-Levin, R.Cervera, M.F.Doran, and M. Dougados. "Vaccination in adult patients with auto-immune inflammatory rheumatic diseases: a systematic literature review for the European League Against Rheumatism evidence-based recommendations for vaccination in adult patients with auto-immune inflammatory rheumatic diseases." Autoimmun Rev.vol. 10,pp.341-52,2011.

[10] M.Bijl, N.Agmon-Levin, J.M.Dayer, E.Israeli, M.Gatto, and Y.Shoenfeld. "Vaccination of patients with auto-immune inflammatory rheumatic diseases requires careful benefit-risk assessment." Autoimmun Rev.vol. 11,pp. 572576,2012 .

[11]C.Rondaan, V.Furer, M.W.Heijstek, N.AgmonLevin, M.Bijl, F.C.Breedveld, O.Elkayam, Efficacy, immunogenicity and safety of vaccination in adult patients with autoimmune inflammatory rheumatic diseases: a systematic literature review for the 2019 update of EULAR recommendations. RMD open.vol. 5(2),pp, e001035,2019.

[12]M.Szwejkowska, E.Kuchar. Vaccinations in rheumatic diseases. Reumatologia.vol. 58(2),pp. 61,2020

[13]T.P.Monath. Review of the risks and benefits of yellow fever vaccination including some new analyses. Expert review of vaccines.vol.11(4),pp. 427-448,2012.

[14]P.K.Wong, H.Bagga, C.Barrett, P.Hanrahan, D.Johnson, A.Katrib, L.Young. A practical approach to vaccination of patients with autoimmune inflammatory rheumatic diseases in Australia. Internal medicine journal.vol.47(5),pp.491-500,2017.

[15] V.Shobha. Common anti-infective prophylaxis and vaccinations in autoimmune inflammatory rheumatic diseases. Indian Journal of Rheumatology.vol. 7(1),pp. 21-28,2012.

[16]L.Szenborn. Vaccinations in autoimmune inflammatory rheumatic diseases. Reumatologia.vol. 54(6),pp. 275,2016.

[17]P.K.Wong, H.Bagga, C.Barrett, P.Hanrahan, D.Johnson, A.Katrib, L.Young. A practical approach to vaccination of patients with autoimmune inflammatory rheumatic diseases in Australia. Internal medicine journal.vol. 47(5),pp. 491-500,2017.

[18] C.Hua, T.Barnetche, B.Combe, J.Morel. Effect of Methotrexate, Anti-Tumor Necrosis Factor $\alpha$, and Rituximab on the Immune Response to Influenza and Pneumococcal Vaccines in Patients With Rheumatoid Arthritis: A Systematic Review and Meta-Analysis. Arthritis care \& research.vol. 66(7),pp. 1016-1026,2014. 
[19]É.Rákóczi, B.Perge, E.Végh, P.Csomor, A.Pusztai, S.Szamosi, Z.Szekanecz. Evaluation of the immunogenicity of the 13-valent conjugated pneumococcal vaccine in rheumatoid arthritis patients treated with etanercept. Joint Bone Spine.vol. 83(6),pp. 675-679,2016.

[20] LS.Tam, PK.Chan, SC.Ho, SC.Ho, MY.Yu, SF.Y im. Natural history of cervical papilloma virus infection in systemic lupus erythematosus - a prospective cohort study. J Rheumatol .vol. 37,pp. 333- 40,2010.

[21]I.H.R.Grein, N.Groot, M.I.Lacerda, N.Wulffraat, G.Pileggi. HPV infection and vaccination in Systemic Lupus Erythematosus patients: what we really should know. Pediatric Rheumatology.vol. 14(1),pp. 1-8,2016.

[22] CO.Bingham, JR.Looney, A.Deodhar, N.Halsey, M.Greenwald, C.Codding .Immunisation responses in rheumatoid arthritis patients treated with rituximab. Arthritis Rheum.vol. 62,pp. 6474,2010 .

[23] V.Furer, C.Rondaan, M.W.Heijstek, N.AgmonLevin, S.Van Assen, M.Bijl, O.Elkayam.update of EULAR recommendations for vaccination in adult patients with autoimmune inflammatory rheumatic diseases. Annals of the rheumatic diseases.vol.79(1),pp. 39-52,2020.

[24] L.Bernini, CU.Manzini, D.Giuggioli, M.Sebastian i, C.Ferri. Reactive arthritis induced by intravesical BCG therapy for bladder cancer: our clinical experience and systematic review of the literature. Autoimmun Rev .vol. 12,pp. 11509,2013 .

[25]I.Ferreira, D.Isenberg. Vaccines and biologics. Annals of the rheumatic diseases.vol.73(8),pp. 1446-1454,2014. 